\title{
Regularity of intuitionistic fuzzy relations on hypergroupoids
}

\section{Irina Cristea}

This article is dedicated to my esteemed PhD supervisor, professor dr. Mirela Ştefănescu, for celebration of her 70th birthday.

\begin{abstract}
In this paper we introduce the notions of regular and strongly regular intuitionistic fuzzy relations on hypergroupoids, studying some related properties and connections with the classical case. Then we investigate the lattice structure of these kinds of intuitionistic fuzzy equivalences.
\end{abstract}

\section{Introduction}

Connections between algebraic hyperstructures and binary relations or fuzzy relations have been established with the aim to obtain new sets with similar hyperoperations as the initial ones. The most well-known constructions of hypergroupoids from binary relations are those proposed by Rosenberg [22], Corsini-Leoreanu [8, 9], Spartalis [24, 25], Cristea-Ştefănescu [10, 11, 12], extended later to $n$-ary hypergroupoids by Davvaz, Leoreanu-Fotea [13], or to actions of hyperstructures by Chvalina et al. [6], or to topological hypergroups by Hoskova [17] . Feng [15] obtained fuzzy hypergroups from fuzzy relations, while Jančić-Rašović in [21] constructed hyperrings from fuzzy relations defined on a semigroup.

Key Words: Intuitionistic fuzzy set; (Strongly) regular intuitionistic fuzzy relation; Hypergroupoid; Distributive lattice

2010 Mathematics Subject Classification: MSC2010: 20N20; 03E72

Received: June 2013

Revised: July 2013

Accepted: September 2013 
Hur et al. $[18,20]$ introduced the intuitionistic fuzzy equivalence relations on lattices and semigroups, studying their properties connected with the binary operations. Like in the classical case, an intuitionistic fuzzy equivalence compatible with the operations is called intuitionistic fuzzy congruence. This kind of relations represents an important tool to obtain classical algebraic structures from similar intuitionistic fuzzy structures, defining a suitable binary operation on the quotient structure obtained modulo the intuitionistic fuzzy congruence. Passing now to the algebraic hyperstructures (where the composition between two elements is a set, and not only an element like in a classical structure), the role of the congruences (classical or fuzzy) is played now by the regular relations (called also congruences by some authors, for example see [3]). Continuing the work of Zhan [26], where he defined and studied the fuzzy regular relations on hyperquasigroups, in this note we introduce the regular intuitionistic fuzzy relations on hypergroupoids. Even if the change of the terminology (with respect to Zhan's paper [26]) could create some confusions, we prefer to do this here, since we study the regularity of the intuitionistic fuzzy relations, that permits to define regular intuitionistic fuzzy relations. Using this convention, the notion introduced in [26] would be regular fuzzy relation, and not fuzzy regular relation. After some characterizations of these type of intuitionistic fuzzy relations, we investigate also their lattice structure. In the last section, some conclusions summarizing the results and some future lines of research are discussed.

\section{Preliminaries concerning intuitionistic fuzzy relations}

In this section we recall some definitions from intuitionistic fuzzy relation theory and we fix the notations used in this paper.

Definition 2.1. [1,2] An intuitionistic fuzzy set (shortly IFS) on a universe $X$ is an object having the form $A=\left\{\left(x, \mu_{A}(x), \nu_{A}(x)\right) \mid x \in X\right\}$, where $\mu_{A}(x) \in[0,1]$, called the degree of membership of $x$ in $A, \nu_{A}(x) \in[0,1]$, called the degree of non-membership of $x$ in $A$, verify, for any $x \in X$, the relation $0 \leq \mu_{A}(x)+\nu_{A}(x) \leq 1$. The class of IFSs on a universe $X$ will be denoted by $\mathcal{J F S}(X)$.

It is clear that an IFS can be considered as a fuzzy set whenever $\nu_{A}(x)=$ $1-\mu_{A}(x)$, for any $x \in X$, but conversely not.

Definition 2.2. $[1,2]$ An intuitionistic fuzzy relation $R$ (shortly IFR) from a universe $X$ to a universe $Y$ is an IFS in $X \times Y$, i.e. a set by the form $R=\left\{\left((x, y) ; \mu_{R}(x, y), \nu_{R}(x, y)\right) \mid(x, y) \in X \times Y\right\}$, where $0 \leq \mu_{R}(x, y)+$ $\nu_{R}(x, y) \leq 1$, for any $(x, y) \in X \times Y$. 
Furthermore, the number $\pi_{R}(x, y)=1-\mu_{R}(x, y)-\nu_{R}(x, y)$, for $(x, y) \in$ $X \times Y$, is called the index of the element $(x, y)$ in IFR $R$ and it is described as a degree of hesitation whether $x$ and $y$ are in the relation $R$ or not.

The class of IFRs from $X$ to $Y$ will be denoted by $\mathcal{J F R}(X \times Y)$ and the class of IFRs on $X$ will be denoted by $\mathcal{J F} \mathcal{R}(X)$.

The domain of $R \in \mathcal{J F} \mathcal{F}(X \times Y)$ is the IFS in $X$ defined by $\operatorname{dom}(R)=$ $\left\{\left(x, \bigvee_{y \in Y} \mu_{R}(x, y), \bigwedge_{y \in Y} \nu_{R}(x, y)\right) \mid x \in X\right\}$ and the range of $R$ is the IFS in $Y$ defined by $\operatorname{rng}(R)=\left\{\left(x, \bigvee_{x \in X} \mu_{R}(x, y), \bigwedge_{x \in X} \nu_{R}(x, y)\right) \mid y \in Y\right\}$.

In the following we mention some basic operations between IFRs. For more details see $[5,14]$.

Definition 2.3. $i)$ Let $R$ and $S$ be in $\mathcal{J F} \mathcal{R}(X \times Y)$. For every $(x, y) \in X \times Y$, we define

1. $R \subseteq S \Longleftrightarrow \mu_{R}(x, y) \leq \mu_{S}(x, y)$ and $\nu_{R}(x, y) \geq \nu_{S}(x, y)$

2. $R \preceq S \Longleftrightarrow \mu_{R}(x, y) \leq \mu_{S}(x, y)$ and $\nu_{R}(x, y) \leq \nu_{S}(x, y)$

3. $R \cup S=\left\{\left((x, y), \mu_{R}(x, y) \vee \mu_{S}(x, y), \nu_{R}(x, y) \wedge \nu_{S}(x, y)\right)\right\}$

4. $R \cap S=\left\{\left((x, y), \mu_{R}(x, y) \wedge \mu_{S}(x, y), \nu_{R}(x, y) \vee \nu_{S}(x, y)\right)\right\}$

5. $R^{c}=\left\{\left((x, y), \nu_{R}(x, y), \mu_{R}(x, y)\right)\right\}$.

The family $(\mathcal{J F R}(X \times Y), \cup, \cap)$ is a complete, distributive lattice, with respect to the partially ordering $\preceq$.

ii) Let $R$ in $\mathcal{J F} \mathcal{R}(X \times Y)$ and $S$ in $\mathcal{J F R}(Y \times Z)$. Then the composition between $R$ and $S$ is an IFR on $X \times Z$ defined as

$$
R \circ S=\left\{\left((x, z), \bigvee_{y \in Y}\left(\mu_{R}(x, y) \wedge \mu_{S}(y, z)\right), \bigwedge_{y \in Y}\left(\nu_{R}(x, y) \vee \nu_{S}(y, z)\right)\right)\right\}
$$

whenever $0 \leq \bigvee_{y \in Y}\left(\mu_{R}(x, y) \wedge \mu_{S}(y, z)\right)+\bigwedge_{y \in Y}\left(\nu_{R}(x, y) \vee \nu_{S}(y, z)\right) \leq 1$.

Now we consider the IFRs defined on a set $X$.

Definition 2.4. An IFR $R$ on a set $X$ is called an intuitionistic fuzzy equivalence relation (on short, IFER) on $X$ if it satisfies the following conditions:

1. it is intuitionistic fuzzy reflexive: $R(x, x)=(1,0)$, for any $x \in X$;

2. it is intuitionistic fuzzy symmetric: $R(x, y)=R(y, x)$, for any $x, y \in X$;

3. it is intuitionistic fuzzy transitive: $R^{2}=R \circ R \subseteq R$. 
Let $R$ be an IFER on $X$ and let $a \in X$. The intuitionistic fuzzy set $R a$ in $X$ defined by $R a(x)=R(a, x)$, for each $x \in X$, is called an intuitionistic fuzzy equivalence class of $R$ containing $a \in X$. The set $\{R a \mid a \in X\}$ is called the intuitionistic fuzzy quotient set of $X$ by $R$ and it is denoted by $X / R$.

Similarly to the classic case, the intuitionistic fuzzy equivalence classes satisfy several basic properties.

Proposition 2.5. [20] Let $R$ be an IFER on a set $X$. Then the following statements hold:

1. $R a=R b$ if and only if $R(a, b)=(1,0)$, for any $a, b \in X$.

2. $R(a, b)=(0,1)$ if and only if $R a \cap R b=\underset{\sim}{0}$, where $\underset{\sim}{0}(x)=(0,1)$, for any $x \in X$.

3. $\bigcup_{a \in X} R a=\underset{\sim}{1}$, where $\underset{\sim}{1}(x)=(1,0)$, for any $x \in X$.

4. There exists the onto application $\pi: X \longrightarrow X / R$ defined by $\pi(x)=R x$, for any $x \in X$.

\section{Regularity of intuitionistic fuzzy relations on hyper- groupoids}

The congruences in a semigroup have been extended to regular and strongly regular relations in a hypergroupoid. They are used to obtain quotient semihypergroups and quotient semigroups, respectively. We recall here these classical notions from [7].

Let $\rho$ be an equivalence relation on a hypergroupoid $H$. If $A$ and $B$ are non-empty subsets of $H$, we write $A \bar{\rho} B$ to denote that, for each $a \in A$, there exists $b \in B$ such that $a \rho b$ and, for each $b \in B$, there exists $a \in A$ such that $a \rho b$. We write $A \overline{\bar{\rho}} B$ if, for each $a \in A$ and $b \in B$, one has $a \rho b$.

Definition 3.1. An equivalence relation $\rho$ on a hypergroupoid $(H, \circ)$ is called regular to the right if, for every $(x, y) \in H^{2}, x \rho y$ implies that $x \circ a \bar{\rho} y \circ a$, for each $a \in H$. The equivalence $\rho$ is called strongly regular to the right if, for every $(x, y) \in H^{2}, x \rho y$ implies that $x \circ a \overline{\bar{\rho}} y \circ a$, for each $a \in H$. Similarly, the (strongly) regularity on the left is defined. A (strongly) regular relation to the right and to the left is called (strongly) regular relation.

The next result expresses the role of these kinds of equivalences in semihypergroups, where the associativity property holds. 
Theorem 3.2. [7] Let $(H, \circ)$ be a semihypergroup and $\rho$ an equivalence on $H$.

1. If $\rho$ is a regular relation, then a semihypergroup structure turns out to be defined on the quotient set $H / \rho$, with respect to the hyperproduct defined by $\rho(x) * \rho(y)=\{\rho(z) \mid z \in x \circ y\}$.

2. Conversely, if the previous hyperoperation is well-defined, and thus $(H / \rho, *)$ is a semihypergroup, then $\rho$ is regular.

3. If $\rho$ is strongly regular, then $(H / \rho, *)$ is a semigroup. Moreover, if $(H, \circ)$ is a hypergroup, then $(H / \rho, *)$ is a group.

The fact that $(H, \circ)$ is a semihypergroup (and not just a hypergroupoid) is essential in the proofs of above three statements.

Following the current trend to provide natural fuzzyfications of crisp concepts, like a theoretical method to deal with imprecision, vagueness, uncertainty, we define the regularity also for intuitionistic fuzzy equivalence relations on a hypergroupoid.

Definition 3.3. An IFER $R$ on a hypergroupoid $(H, \circ)$ is said to be

1. intuitionistic fuzzy regular on the left if, for any $x, y, z \in H$, and for any $u \in z \circ x$, there exists $v \in z \circ y$ such that

$$
\mu_{R}(x, y) \leq \mu_{R}(u, v), \quad \nu_{R}(x, y) \geq \nu_{R}(u, v)
$$

and, for any $v \in z \circ y$, there exists $u \in z \circ x$ such that (R) holds.

2. intuitionistic fuzzy regular on the right if, for any $x, y, z \in H$, and, for any $u \in x \circ z$, there exists $v \in y \circ z$ such that relation (R) holds, and for any $v \in y \circ z$, there exists $u \in x \circ z$ such that relation (R) holds, too.

3. intuitionistic fuzzy regular, more exactly intuitionistic fuzzy totally regular if, for any $x, y, z, t \in H$, and for all $u \in x \circ z$, there exists $v \in y \circ t$ such that

$$
\mu_{R}(x, y) \wedge \mu_{R}(z, t) \leq \mu_{R}(u, v), \quad \nu_{R}(x, y) \vee \nu_{R}(z, t) \geq \nu_{R}(u, v)
$$

and, for all $v \in y \circ z$, there exists $u \in x \circ z$ such that relation (RR) holds.

Remark 3.4. We can notice the difference between the two cases (crisp and intuitionistic fuzzy equivalences), that is the generality of the regularity property in the intuitionistic fuzzy case. More exactly, if $R$ is an intuitionistic fuzzy relation regular on the left, then it satisfies also the property of a crisp relation regular on the left, i.e.: $R x=R y$ implies that, for any $z \in H$ and any 
$u \in z \circ x$, there exists $v \in z \circ y$ such that $R u=R v$. The same property is valid also for intuitionistic fuzzy relation regular on the right, or just regular.

Indeed, $R x=R y$ is equivalent with $\mu_{R}(x, y)=1$ and $\nu_{R}(x, y)=0$. Since $R$ is intuitionistic fuzzy regular on the left, it follows that, for any $u \in z \circ x$, there exists $v \in z \circ y$ such that $1=\mu_{R}(x, y) \leq \mu_{R}(u, v)$ and $0=\nu_{R}(x, y) \geq \nu_{R}(u, v)$, i.e. $\mu_{R}(u, v)=1$ and $\nu_{R}(u, v)=0$. Thus $R u=R v$.

The connections between left, right and totally regularity are described here below.

Lemma 3.5. Let $R$ be an IFER on a hypergroupoid $(H, \circ)$. Then the following conditions are equivalent:

i) $R$ is intuitionistic fuzzy regular.

ii) $R$ is intuitionistic fuzzy regular on the left and on the right.

Proof. Consider $R$ a regular intuitionistic fuzzy relation on $H$. Let $x, y, z \in H$. Since $R$ is intuitionistic fuzzy reflexive, it follows that $R(z, z)=(1,0)$, that is $\mu_{R}(z, z)=1$ and $\nu_{R}(z, z)=0$. According with the hypothesis, we get that, for any $u \in x \circ z$, there exists $v \in y \circ z$ such that $\mu_{R}(x, y)=\mu_{R}(x, y) \wedge \mu_{R}(z, z) \leq$ $\mu_{R}(u, v)$ and $\nu_{R}(x, y)=\nu_{R}(x, y) \vee \nu_{R}(z, z) \geq \nu_{R}(u, v)$.

Thus, we proved that $R$ is intuitionistic fuzzy regular on the right. Similarly, we can obtain that $R$ is also intuitionistic fuzzy regular on the left.

Conversely, suppose that $R$ is intuitionistic fuzzy regular on the left and on the right. Let $x, y, z, t \in H$. Then, for any $u \in x \circ z$, there exists $w \in y \circ z$ such that $\mu_{R}(x, y) \leq \mu_{R}(u, w)$ and $\nu_{R}(x, y) \geq \nu_{R}(u, w)$. Now, for $w \in y \circ z$, there exists $v \in y \circ t$ such that $\mu_{R}(z, t) \leq \mu_{R}(w, v)$ and $\nu_{R}(z, t) \geq \nu_{R}(w, v)$.

Thereby, since $R$ is intuitionistic fuzzy transitive, it follows that, for any $u \in x \circ z$, there exists $v \in y \circ t$ such that $\mu_{R}(x, y) \wedge \mu_{R}(z, t) \leq \mu_{R}(u, w) \wedge$ $\mu_{R}(w, v) \leq \mu_{R}(u, v)$, so $R$ is intuitionistic fuzzy regular, too.

Definition 3.6. An IFER $R$ on a hypergroupoid $(H, \circ)$ is called

1. intuitionistic fuzzy strongly regular on the left if, for any $x, y, z \in H$,

$$
\begin{gathered}
\mu_{R}(x, y) \leq \inf \left\{\mu_{R}(u, v) \mid u \in z \circ x, v \in z \circ y\right\} \\
\nu_{R}(x, y) \geq \sup \left\{\nu_{R}(u, v) \mid u \in z \circ x, v \in z \circ y\right\} .
\end{gathered}
$$

2. intuitionistic fuzzy strongly regular on the right if, for any $x, y, z \in H$,

$$
\begin{aligned}
& \mu_{R}(x, y) \leq \inf \left\{\mu_{R}(u, v) \mid u \in x \circ z, v \in y \circ z\right\}, \\
& \nu_{R}(x, y) \geq \sup \left\{\nu_{R}(u, v) \mid u \in x \circ z, v \in y \circ z\right\} .
\end{aligned}
$$


3. intuitionistic fuzzy strongly regular if, for any $x, y, z, t \in H$,

$$
\begin{aligned}
& \mu_{R}(x, y) \wedge \mu_{R}(z, t) \leq \inf \left\{\mu_{R}(u, v) \mid u \in x \circ z, v \in y \circ z\right\}, \\
& \nu_{R}(x, y) \vee \nu_{R}(z, t) \geq \sup \left\{\nu_{R}(u, v) \mid u \in x \circ z, v \in y \circ z\right\} .
\end{aligned}
$$

Similarly with Lemma 3.5, we obtain the following characterization.

Lemma 3.7. Let $R$ be an IFER on a hypergroupoid $(H, \circ)$. Then the following conditions are equivalent:

i) $R$ is intuitionistic fuzzy strongly regular.

ii) $R$ is intuitionistic fuzzy strongly regular on the left and on the right.

Example 3.8. Let's consider the identity IFER $R=\Delta[20]$ defined on a nonempty set $H$ by:

$$
\Delta(x, y)= \begin{cases}(1,0) & \text { if } x=y \\ (1,0) & \text { if } x \neq y\end{cases}
$$

Then $\Delta$ is intuitionistic fuzzy regular on any quasihypergroup $(H, \circ)$. Indeed, let $x, y, z, t$ be arbitrary elements in $(H, \circ)$. If $x=y$, then $\mu_{R}(x, y)=1$ and $\nu_{R}(x, y)=0$. Moreover, if $z=t$, then $\mu_{R}(z, t)=1$ and $\nu_{R}(z, t)=0$. Thus, for any $u \in x \circ z$, there exists $v=u \in y \circ t$ such that the relation (RR) in Definition 3.3 holds. If $z \neq t$, then $\mu_{R}(z, t)=0$ and $\nu_{R}(z, t)=1$; therefore $0=\mu_{R}(x, y) \wedge \mu_{R}(z, t) \leq \mu_{R}(u, v)$ and $1=\nu_{R}(x, y) \vee \nu_{R}(z, t) \geq \nu_{R}(u, v)$, for any $v \in y \circ t$ ( since $H$ is a quasihypergroup, the hyperproduct $y \circ t$ is always nonempty). Again the relation (RR) holds. Finally, if $x \neq y$, then $0=\mu_{R}(x, y) \wedge \mu_{R}(z, t) \leq \mu_{R}(u, v)$ and $1=\nu_{R}(x, y) \vee \nu_{R}(z, t) \geq \nu_{R}(u, v)$, for any $v \in y \circ t$.

What can we say about the strongly regularity of $\Delta$ ? We know that $\Delta$ is intuitionistic fuzzy strongly regular on any groupoid [19], but not on any hypergroupoid, as we can see here below. If in the relation (SR) in Definition 3.6 , we take $x=y$ and $z=t$, we have to prove that $1 \leq \mu_{R}(u, v)$, for any $u, v \in x \circ z$, which is false for $u \neq v$. So $\Delta$ is not intuitionistic fuzzy strongly regular for any hypergroupoid.

Example 3.9. On the quasihypergroup $\left(H_{1}, \circ\right)$ represented by the following table:

\begin{tabular}{c|c|c|c}
$H_{1}$ & 1 & 2 & 3 \\
\hline 1 & 1,3 & $H$ & 1,3 \\
\hline 2 & $H$ & 2 & 2,3 \\
\hline 3 & 1,3 & 2,3 & 3
\end{tabular}


consider the IFER $R$ defined as follows:

$$
\begin{array}{ll}
\mu_{R}(1,1)=1 & \nu_{R}(1,1)=0 \\
\mu_{R}(1,2)=1 & \nu_{R}(1,2)=0 \\
\mu_{R}(1,3)=0.7 & \nu_{R}(1,3)=0.1 \\
\mu_{R}(2,2)=1 & \nu_{R}(2,2)=0 \\
\mu_{R}(2,3)=0.4 & \nu_{R}(2,3)=0.3 \\
\mu_{R}(3,3)=1 & \nu_{R}(3,3)=0 .
\end{array}
$$

We show that $R$ is not intuitionistic fuzzy regular on the left, and thus either on the right, since $(H, \circ)$ is commutative. Take $x=1, y=2$ and $z=2$. Then for $u=3 \in 2 \circ 1=z \circ x$, there exists only one element in $z \circ y=2 \circ 2$, namely $v=2$, such that $\mu_{R}(u, v)=\mu_{R}(3,2)=\mu_{R}(2,3)=0.4$, which does not satisfy relation $(\mathrm{R})$ in Definition 3.3, since $0.4=\mu_{R}(u, v) \leq \mu_{R}(x, y)=1$.

Example 3.10. Consider now the same IFER $R$ like in Example 3.9 on the quasihypergroup $\left(H_{2}, \circ\right)$ represented by the table:

\begin{tabular}{c|c|c|c}
$H_{2}$ & 1 & 2 & 3 \\
\hline 1 & 1,2 & 1,2 & $H$ \\
\hline 2 & 1,2 & 1,2 & $H$ \\
\hline 3 & 3 & 3 & 3
\end{tabular}

It is not difficult to verify that $R$ is intuitionistic fuzzy regular on the left, but not on the right. For example, taking $x=1, y=3$, and $z=1$, for $u=2 \in 1 \circ 1=x \circ z$, there exists only one $v=3 \in z \circ 1=y \circ z$, for which we have $\mu_{R}(x, y)=0.7$ and $\mu_{R}(u, v)=0.4$. Therefore $\mu_{R}(x, y) \geq \mu_{R}(u, v)$, that is relation $(\mathrm{R})$ in Definition 3.3 is not valid.

Moreover, in a similar way, one can prove that $R$ neither is intuitionistic fuzzy strongly regular on the left.

On the other side, the regular intuitionistic fuzzy relations on a hypergroupoid can be characterized by the meaning of the upper and lower $\alpha$-cuts. First we recall their definitions from $[4,16,23]$.

Definition 3.11. i) For any $\alpha \in[0,1]$ and a fuzzy relation $\mu$ on a nonempty set $X$, the set

$$
U(\mu, \alpha)=\{(x, y) \in X \times X \mid \mu(x, y) \geq \alpha\}
$$

is called the upper $\alpha$-cut of $\mu$, and the set

$$
L(\mu, \alpha)=\{(x, y) \in X \times X \mid \mu(x, y) \leq \alpha\}
$$

is called the lower $\alpha$-cut of $\mu$. 
ii) For any $\alpha, \beta \in[0,1], \alpha+\beta \leq 1$, and an IFR $R=\left(\mu_{R}, \nu_{R}\right)$ on $X$, the set

$$
C_{\alpha, \beta}(R)=\left\{(x, y) \in X \times X \mid \mu_{R}(x, y) \geq \alpha, \nu_{R}(x, y) \leq \beta\right\}
$$

is called the $(\alpha, \beta)$-cut of $R$.

Now we are able to characterize the intuitionistic fuzzy (strongly) regularity of the intuitionistic fuzzy relations by the meaning of the (strongly) regularity of the associated cuts.

Proposition 3.12. Let $R=\left(\mu_{R}, \nu_{R}\right)$ be an IFER on a hypergroupoid $(H, \circ)$.

i) Then $R$ is intuitionistic fuzzy regular if and only if, for all $\alpha, \beta \in[0,1]$, the cuts $U\left(\mu_{R}, \alpha\right)$ and $L\left(\nu_{R}, \beta\right)$ are regular crisp relations on $H$.

ii) Moreover, $R$ is intuitionistic fuzzy regular if and only if, for all $\alpha, \beta \in$ $[0,1]$, with $\alpha+\beta \leq 1$, the $(\alpha, \beta)$-cut $C_{\alpha, \beta}(R)$ is a regular crisp relation on $H$.

Proof. i) Suppose that $R$ is a regular intuitionistic fuzzy relation on a hypergroupoid $(H, \circ)$. Let $\alpha, \beta \in[0,1]$. Accordingly with Theorem 3.6 [16], the cuts $U\left(\mu_{R}, \alpha\right)$ and $L\left(\nu_{R}, \beta\right)$ are equivalence relations on $H$.

We prove now that $U\left(\mu_{R}, \alpha\right)$ is regular on $H$. Take $x, y \in H$ such that $(x, y) \in U\left(\mu_{R}, \alpha\right)$ and let $a \in H$. By the intuitionistic fuzzy regularity of $R$, for any $u \in x \circ a$, there exists $v \in y \circ a$ such that $\mu_{R}(x, y) \wedge \mu_{R}(a, a) \leq \mu_{R}(u, v)$, equivalently with $\alpha \leq \mu_{R}(x, y) \leq \mu_{R}(u, v)$, that is $(u, v) \in U\left(\mu_{R}, \alpha\right)$, so $U\left(\mu_{R}, \alpha\right)$ is regular. Similarly, $L\left(\nu_{R}, \beta\right)$ is regular on $H$, too.

Conversely, suppose that, for any $\alpha, \beta \in[0,1]$, the cuts $U\left(\mu_{R}, \alpha\right)$ and $L\left(\nu_{R}, \beta\right)$ are regular crisp relations on $H$. Again, accordingly with Theorem $3.6[16], R=\left(\mu_{R}, \nu_{R}\right)$ is an IFER on $H$. It remains to prove that $R$ is intuitionistic fuzzy regular on $H$.

Let $x, y, z, t$ be arbitrary elements in $H$. Suppose that $\mu_{R}(x, y)=s$ and $\mu_{R}(z, t)=s^{\prime}$. Take $\alpha=s \wedge s^{\prime}$. Then $(x, y),(z, t) \in U\left(\mu_{R}, \alpha\right)$. By the regularity of the $\alpha$-cut $U\left(\mu_{R}, \alpha\right)$, for any $u \in x \circ z$, there exists $u^{\prime} \in y \circ z$ such that $\left(u, u^{\prime}\right) \in U\left(\mu_{R}, \alpha\right)$. Then, for $u^{\prime} \in y \circ z$, there exists $v \in y \circ t$ such that $\left(u^{\prime}, v\right) \in U\left(\mu_{R}, \alpha\right)$. Using the transitivity property of $U\left(\mu_{R}, \alpha\right)$, we obtain that, for any $u \in x \circ z$, there exists $v \in y \circ t$ such that $(u, v) \in U\left(\mu_{R}, \alpha\right)$. Therefore, $\mu_{R}(u, v) \geq \alpha=s \wedge s^{\prime}=\mu_{R}(x, y) \wedge \mu_{R}(z, t)$.

Similarly, using the regularity and transitivity property of the $\beta$-cut $L\left(\nu_{R}, \beta\right)$, we obtain that, for any $u \in x \circ z$, there exists $v \in y \circ t$ such that $\nu_{R}(u, v) \leq \nu_{R}(x, y) \vee \nu_{R}(z, y)$, which completes the proof of the fact that the IFR $R$ is intuitionistic fuzzy regular on $H$.

ii) The proof is similar to the previous case and it is based on Theorem 3.7 [4], stating that $R$ is IFER on $H$ if and only if $C_{\alpha, \beta}(R)$ is an equivalence on $H$, with $0 \leq \alpha, \beta \leq 1$ and $\alpha+\beta \leq 1$. 
We conclude this section with two fundamental results, which are similar to Theorem 3.2 in the classical (crisp) case.

Theorem 3.13. Let $R$ be a regular intuitionistic fuzzy relation on a semihypergroup $(H, \circ)$. Define the hyperoperation $*$ on the quotient set $H / R$ as follows: for any $x, y \in H$,

$$
R x * R y=\{R z \mid z \in x \circ y\} .
$$

1. The hyperoperation $*$ is well-defined and $(H / R, *)$ is a semihypergroup.

2. In the above hypothesis, the projection $\pi: H \longrightarrow H / R$ is a good epimorphism of semihypergroups. Moreover, if $(H, \circ)$ is a hypergroup, then the quotient $(H / R, *)$ is a hypergroup, too.

Proof. 1. Let $x, x^{\prime}, y, y^{\prime}$ be in $H$ such that $R x=R x^{\prime}$ and $R y=R y^{\prime}$. It is necessary to prove that $R x^{\prime} * R y^{\prime}=R x * R y$.

Let $R w$ be arbitrary in $R x^{\prime} * R y^{\prime}$, thus $w \in x^{\prime} \circ y^{\prime}$. By the intuitionistic fuzzy regularity of $R$, there exists $u \in x \circ y^{\prime}$ such that $\mu_{R}\left(x, x^{\prime}\right) \leq \mu_{R}(w, u)$ and $\nu_{R}\left(x, x^{\prime}\right) \geq \nu_{R}(w, u)$. Since $R x=R x^{\prime}$, it follows that $\mu_{R}\left(x, x^{\prime}\right)=1$ and $\nu_{R}\left(x, x^{\prime}\right)=0$, and therefore $\mu_{R}(w, u)=1$ and $\nu_{R}(w, u)=0$, which means that $R(w, u)=(1,0)$, equivalently with $R w=R u$.

Similarly, for $u \in x \circ y^{\prime}$, there exists $v \in x \circ y$ such that $R u=R v$.

Concluding, for any $w \in x^{\prime} \circ y^{\prime}$, there exists $v \in x \circ y$ such that $R w=$ $R v \in R x * R y$, thereby $R x^{\prime} * R y^{\prime} \subseteq R x * R y$. In a similar way, one proves the converse inclusion, so the statement is valid.

Now we prove the associativity of the hyperoperation *. Let $R x, R y, R z$ be classes in $H / R$ and $R w \in(R x * R y) * R z$. It means that there exists $R u \in R x * R y$, i.e. $u \in x \circ y$, such that $R w \in R u * R z$, i.e. $w \in u \circ z$. By the associativity of the hyperoperation $\circ$, it follows that $w \in(x \circ y) \circ z=$ $x \circ(y \circ z)$. Thus, there exists $v \in y \circ z$ such that $w \in x \circ v$, equivalently with $R w \in R x *(R y * R z)$, i.e. $(R x * R y) * R z \subseteq R x *(R y * R z)$. Similarly one proves the converse inclusion, which means that the hyperproduct $*$ is associative.

2. It is obvious that the projection $\pi$ is a good epimorphism, that is, for any $x, y \in H, \pi(x \circ y)=\pi(x) * \pi(y)$.

Finally, if $(H, \circ)$ is a hypergroup, from its reproducibility we obtain that $\pi(H) * \pi(x)=\pi(H \circ x)=\pi(H)=\pi(x) * \pi(H)$, thus $(H / R, *)$ is a hypergroup.

Remark 3.14. The converse statement of the first part of the previous theorem is not valid like in the crisp case. Indeed, if the hyperoperation $*$ is well-defined, then it is not necessary that $R$ be a regular intuitionistic fuzzy relation. 
For example, consider the IFER $R$ on the quasihypergroup $\left(H_{2}, \circ\right)$ defined in Example 3.10. We have proven that $R$ is not intuitionistic fuzzy regular on the right. Here we show that the induced hyperoperation $*$ on the quotient set $H / R$ introduced in Theorem 3.13 is well-defined. Taking $x=1$ and $x^{\prime}=2$, it follows that $R x=R x^{\prime}$ (since $\mu_{R}(1,2)=1$ and $\left.\nu_{R}(1,2)=0\right)$. We need to prove that, for any $y \in H$, we obtain $R 1 * R y=R 2 * R y$ and $R y * R 1=R y * R 2$, for any $y \in H$. Set $y=1$. Then $R 1 * R 1=R 2 * R 1=R 1$ and $R 1 * R 1=R 1 * R 2=R 1$. For $y=2$, we get $R 1 * R 2=R 2 * R 2=R 1$ and $R 2 * R 1=R 2 * R 2=R 1$. Finally, for $y=3$, it follows that $R 1 * R 3=R 2 * R 3=\{R 1, R 3\}$ and $R 3 * R 1=$ $R 3 * R 3=R 3$. So, $*$ is well-defined.

The following theorem connects (semi)hypergroups to (semi)groups through the intuitionistic fuzzy strongly regular relations.

Theorem 3.15. Let $R$ be a strongly regular intuitionistic fuzzy relation on a semihypergroup $(H, \circ)$. Then

1. $(H / R, *)$ is a semigroup.

2. If $(H, \circ)$ is a hypergroup, then the quotient $(H / R, *)$ is a group.

Proof. 1. It is enough to prove that, for any $x, y \in H$, we have $|R x * R y|=1$, that is, for any $z_{1}, z_{2} \in x \circ y, R z_{1}=R z_{2}$. Indeed, since $R$ is intuitionistic fuzzy strongly regular, for any $z_{1}, z_{2} \in x \circ y$, it follows that $\mu_{R}(x, x) \wedge \mu_{R}(y, y)=$ $1 \leq \mu_{R}\left(z_{1}, z_{2}\right)$ and $\nu_{R}(x, x) \vee \nu_{R}(y, y)=0 \geq \nu_{R}\left(z_{1}, z_{2}\right)$, which implies that $R\left(z_{1}, z_{2}\right)=(1,0)$, that is $R z_{1}=R z_{2}$.

2. It follows immediately from 1 .

\section{Lattice structure of regular intuitionistic fuzzy rela- tions on a hypergroupoid}

The purpose of this section is to prove that the set of all intuitionistic fuzzy regular relations on a hypergroupoid forms a distributive lattice. For this, we use, firstly, some results regarding the intuitionistic fuzzy equivalences established by Hur et al. [18, 20] and by Basnet-Sarma [4].

Proposition 4.1. [4] If $R$ and $S$ are IFERs on a set $X$, then their intersection $R \cap S$ is an IFER on $X$, too.

The property to be IFER is conserved by the composition between IFRs under a certain condition, expressed in the next result.

Proposition 4.2. [20] If $R$ and $S$ are IFERs on a set $X$ such that $R \circ S=$ $S \circ R$, then the composition $R \circ S$ is an IFER on $X$, too. 
The same properties are conserved also for the intuitionistic fuzzy regular relations.

Proposition 4.3. If $R$ and $S$ are intuitionistic fuzzy regular relations on a hypergroupoid $(H, \circ)$ such that $R \circ S=S \circ R$, then $R \circ S$ and $R \cap S$ are intuitionistic fuzzy regular relations on $H$.

Proof. By Proposition 3.12, it is enough to show that the cuts $U\left(\mu_{R \circ S}, \alpha\right)$, $L\left(\nu_{R \circ S}, \beta\right)$ and respectively $U\left(\mu_{R \cap S}, \alpha\right), L\left(\nu_{R \cap S}, \beta\right)$, are regular relations on $H$.

Like in the fuzzy case [3], it is clear that

$$
\begin{gathered}
U\left(\mu_{R \circ S}, \alpha\right)=U\left(\mu_{R}, \alpha\right) \circ U\left(\mu_{S}, \alpha\right), \\
U\left(\mu_{R \cap S}, \alpha\right)=U\left(\mu_{R}, \alpha\right) \cap U\left(\mu_{S}, \alpha\right), \\
L\left(\nu_{R \circ S}, \beta\right)=L\left(\nu_{R}, \alpha\right) \circ L\left(\nu_{S}, \alpha\right), \\
L\left(\nu_{R \cap S}, \beta\right)=L\left(\nu_{R}, \beta\right) \cap L\left(\nu_{S}, \beta\right),
\end{gathered}
$$

for any $\alpha, \beta \in[0,1]$.

Furthermore, since $R$ and $S$ are intuitionistic fuzzy regular relations on $H$, then by Proposition 3.12, it follows that $U\left(\mu_{R}, \alpha\right), U\left(\mu_{S}, \alpha\right)$ and $L\left(\nu_{R}, \beta\right)$, $L\left(\nu_{S}, \beta\right)$ are regular relations on $H$. Accordingly with Theorem 3.16 [3], the composition of regular relations having the property that the composition is commutative, is a regular relation, and therefore the cuts $U\left(\mu_{R \circ S}, \alpha\right)$, $L\left(\nu_{R \circ S}, \beta\right)$ are regular relations on $H$.

Now we can conclude with the main result of this section.

Theorem 4.4. The set of all intuitionistic fuzzy regular relations on $H$ forms a distributive lattice with respect to the intersection and the composition between such relations.

Proof. If $R, S$ and $T$ are IFERs on $H$, then $R \cap(S \circ T)=(R \cap S) \circ(R \cap T)$, as it was shown in Theorem 3.19 [3].

\section{Conclusions and future work}

In this paper, we have introduced and characterized the regular and strongly regular intuitionistic fuzzy relations on a hypergroupoid, like a generalization of the corresponding classical (crisp) relations. Based on the similar results for fuzzy congruences on hypergroupoids [3], we have proved that the set of all regular intuitionistic fuzzy relations on a hypergroupoid forms a distributive lattice with respect to the intersection and the composition between such relations. 
The case of the strongly regular intuitionistic fuzzy relations (which are representative only for the hyperstructures, since a regular and a strongly regular relation in a classical structure have the same meaning) will be discussed in a future work. Furthermore, extensions of these relations to the fuzzy hypergroupoids will be also investigated. In this case, starting from an equivalence relation defined on a fuzzy hypergroupoid, the notion of intuitionistic fuzzy (strongly) regular relation will be introduced.

Acknowledgements. I wish to express my gratitude to Assoc. Prof. dr. Cristina Flaut, for the organizing of the Workshop "A new approach in theoretical and applied methods in algebra and analysis"Constanta, Romania 4-6 April 2013, where this paper was first presented.

This work was partially supported by "Agencija za raziskovalno dejavnost Republike Slovenije-program P1 - 0285", CNCS - UEFISCDI nr. PN II-IDWE-2012-4-161, and CNCS-UEFISCDI grant PN-II-ID-WE-2012-4-169.

\section{References}

[1] Atanassov, K., Intuitionistic fuzzy sets, Fuzzy Sets and Systems, 20(1986), 87-96.

[2] Atanassov, K., Intuitionistic fuzzy sets: Theory and applications, Heilderberg:Physica-Verlag, 1999.

[3] Bakhshi, M., Borzooei, R.A., Lattice structure on fuzzy congruence relations of a hypergroupoid, Inform. Sci., 177(2007), 3305-3313.

[4] Basnet, D.K., Sarma, N.K., A note on intuitionistic fuzzy equivalence relation, Int. Math. Forum, 67(5)(2010), 3301-3307.

[5] Bustince, H., Burillo, P., Structures on intuitionistic fuzzy relations, Fuzzy Sets and Systems, 78(1996), 293-303.

[6] Chvalina, J., Hoskova-Mayerova, S., Nezhad, A.D., General actions of hyperstructures and some applications, An. Ştiinţ. Univ. Ovidius Constanţa Ser. Mat., 21(1)(2013), 59-82.

[7] Consini, P., Prolegomena of Hypergroup Theory, Aviani Editore, 1993.

[8] Corsini, P., On the hypergroups associated with binary relations, Multi. Val. Logic, 5(2000), 407-419.

[9] Corsini, P., Leoreanu, V., Hypergroups and binary relations, Algebra Universalis, 43(2000), 321-330. 
[10] Cristea, I., Ştefănescu, M., Binary relations and reduced hypergroups, Discrete Math., 308(2008), 3537-3544.

[11] CRisteA, I., Several aspects on the hypergroups associated with n-ary relations, An. Ştiinţ. Univ. Ovidius Constanţa Ser. Mat., 17(3)(2009), 99-110.

[12] CRistea, I., Ştefănescu, M., Hypergroups and n-ary relations, European J. Combin., 31(2010), 780-789.

[13] Davvaz, B., Leoreanu-Fotea, V., Binary relations on ternary semihypergroups, Comm. Algebra, 38(10)(2010), 3621-3636.

[14] Deschrijver, G., Kerre, E., On the composition of the intuitionistic fuzzy relations, Fuzzy Sets and Systems, 136(2003), 333-361.

[15] FenG, Y., p-fuzzy quasi-hypergroups obtained from fuzzy binary relations, J. Discrete Math. Sci. Cryptogr. 13(3)(2010), 201-208.

[16] Ghorbani, S., Intuitionistic fuzzy congruence relations on residuated lattices, Acta Univ. Apulensis Math. Inform., 29(2012), 301-314.

[17] Hoskova-Mayerova, S., Topological hypergroups, Comput. Math. Appl. 64(9)(2012), 2845-2849.

[18] Hur, K., Jang, S.Y., Kang, H.W., Some intuitionistic fuzzy congruences, Iran. J. Fuzzy Syst., 3(1)(2006), 45-57.

[19] Hur, K., Jang, S.Y., Kang, H.W., The lattice of intuitionistic fuzzy congruences, Int. Math. Forum, 5(1)(2006), 211-236.

[20] Hur, K., JAng, S.Y., Ahn, Y.S., Intuitionistic fuzzy equivalence relations, Honam Math. J., 27(2)(2005), 159-177.

[21] JANČIĆ-RAŠOvić, S., On hyperrings associated with $\mathcal{L}$-fuzzy relations, Mathematica Montisnigri, vol. XXIV-XXV, 2011-2012.

[22] Rosenberg, I.G., Hypergroups and join spaces determined by relations, Ital. J. Pure Appl. Math., 4(1998), 93-101.

[23] Sharma, P.K., $(\alpha, \beta)$-Cut of intuitionistic fuzzy groups, Int. Math. Forum, 53(6)(2011), 2605-2614.

[24] Spartalis, S., Mamaloukas, C., On hyperstructures associated with binary relations, Comput. Math. Appl., 51(1)(2006), 41-50. 
[25] Spartalis, S., Konstantinidou-Serafimidou, M., Taouktsoglou, A., C-hypergroupoids obtained by special binary relations, Comput. Math. Appl., 59(2010), 2628-2635.

[26] Zhan, J., Fuzzy Regular Relations on Hyperquasigroups, J. Math. Res. Exposition, 30(6)(2010), 1083-1090.

Irina Cristea,

Centre for Systems and Information Technologies,

University of Nova Gorica, SI-5000, Vipavska 13, Nova Gorica, Slovenia

Email: irinacri@yahoo.co.uk 